\title{
Prospects for Reliable 3D Imaging in Aberration-corrected STEM, TEM and SCEM
}

\author{
Huolin L. Xin, * and David A. Muller** \\ * Department of Physics, Cornell University, Ithaca, NY 14853 \\ ** School of Applied and Engineering Physics, Cornell University, Ithaca, NY 14853
}

The development of aberration correctors allows the illumination angles to be increased, which in turn, greatly shrinks the depth of focus (DoF) of a scanning transmission electron microscope (STEM). The short DoF $(\sim 3-6 \mathrm{~nm})$ could potentially enable the reconstruction of the underlying 3D structures by acquiring a series of through-focal images. To eliminate the channeling artifacts in the depth sectioning of crystalline materials [1], the hollow-cone illuminated STEM (HCI-STEM) [2] was re-examined due to its capability to suppress coupling to the channeling states [3]. In addition to STEM depth sectioning, some efforts have been dedicated to the development of scanning confocal electron microscopes (SCEMs) in hopes of further improving the depth resolution $[4,5]$. To examine the reliability and performance limit of these 3D imaging modes, we have derived the boundaries of their corresponding 3D contrast transfer functions (CTFs) in analytic forms $[6,7]$, and confirmed our theoretical findings in both real and reciprocal space by depth-sectioning experiments.

Sketches of the analytic boundaries of the 3D CTFs of ADF-STEM, BF-STEM/TEM, HCISTEM, coherent BF-SCEM and incoherent SCEM are illustrated in Fig. 1. It is shown that BFSTEM/TEM (Fig. 1a) only transfers information on the two parabolic surfaces (a manifestation of the Ewald sphere). With partial coherence included, the two surfaces will be smeared out, allowing more information to be transferred -- not enough, however, for a reliable 3D reconstruction. On the other hand, the BF-STEM/TEM effectively has a very large DoF ideal for tilt-series or discrete tomography, whereas ADF-STEM tomography is limited by the beam divergence [8] when the sample is much thicker than the probe's DoF. The information transfer of ADF-STEM, BF-SCEM and HCI-STEM depth sectioning are problematic as well. Intuitively, we might expect depth-sectioning reconstructions to be the original objects blurred out by $\sim 3-6 \mathrm{~nm}$ in $\mathrm{z}$-direction. However it is shown in Fig. $1 \mathrm{~b}$ that the CTF has a missing cone problem which results in almost all features being proportionally elongated by the same factor in depth-sectioning reconstructions, independent of their original size. Additionally, BF-SCEM yields no improvement in resolution over ADF-STEM in all three dimensions. Since the opening angle of the $\mathrm{CTF}$ can be shown to be exactly equal to the probe-forming angle $\left(\alpha_{\max }\right)[6,7]$, we find that the elongation factor for ADF-STEM and BF-SCEM is equal to $\sqrt{3 / 2} / \alpha_{\max }$ to first order. For current aberration-corrected STEMs, the probe-forming aperture can only be increased to $40 \mathrm{mrad}$ in the best case, which results in an elongation factor of roughly 30 times. This is potentially dangerous if one interprets the reconstructions without a priori knowledge of the underlying artifacts. In contrast, incoherent SCEM is the only mode of those investigated in which the missing cone is filled (Fig. 1c). As shown in Fig. 2e, by filling the missing cone in reciprocal space, the incoherent SCEM reconstruction closely reproduces the features of the original object, while other imaging modes stretch the original 30-nm object to over 200nm, rendering the reconstructions unrecognizable (Fig. 2b-d).

In order to understand the missing-cone problem experimentally, a 33-mrad $100 \mathrm{KeV}$ C5corrected Nion UltraSTEM was used to take ADF-STEM through-focal series. Fig. 3a shows the central image of a $6 \mathrm{~nm}$ gold particle taken from a through-focal series of a total of 101 images acquired at $4 \mathrm{~nm}$ defocus intervals. It is found that the $\sim 6 \mathrm{~nm}$ gold particle is blurred into a $220 \mathrm{~nm}$ long stick in the reconstruction (Fig. 3b) yielding an elongation factor of approximately 35-40, in close agreement with the theoretically predicted value 37.5 . The missing cone in the CTF is clearly shown in the 3D diffractogram of the depth-sectioning reconstruction (Fig. 3e), where the opening angle is measured to be equal to the convergent angle of the microscope. Moreover, as the gold particle is 
approximately round, the fringe envelope observed in the diffractogram can be understood as the 3D Fourier transform of a solid sphere [9].

\section{References}

[1] H. L. Xin, V. Intaraprasonk, and D. A. Muller, Applied Physics Letters 92, 013125 (2008).

[2] R. F. Loane, and J. Silcox, Proceedings of the 47th Annual Meeting of EMSA, 124 (1989).

[3] H. L. Xin, V. Intaraprasonk, and D. A. Muller, Microscopy and Microanalysis 14, 926 (2008).

[4] P. D. Nellist et al., Microscopy and Microanalysis 14, 104 (2008).

[5] M. Takeguchi et al., J Electron Microsc (Tokyo) 57, 123 (2008).

[6] V. Intaraprasonk, H. L. Xin, and D. A. Muller, Ultramicroscopy 108, 1454 (2008).

[7] H. L. Xin, and D. A. Muller, J Electron Microsc (Tokyo), doi:10.1093/jmicro/dfn029 (2009).

[8] J. K. Hyun, P. Ercius, and D. A. Muller, Ultramicroscopy 109, 1 (2008).

[9] Research supported by Semiconductor Research Corporation and NSF MRSEC DMR\# 0520404.

(a)

(b)

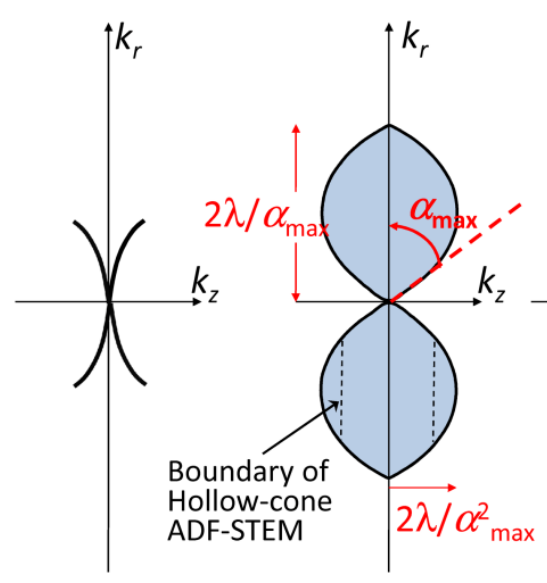

TEM/BF-STEM

\begin{abstract}
ADF-STEM
\end{abstract}
Coherent SCEM (c)

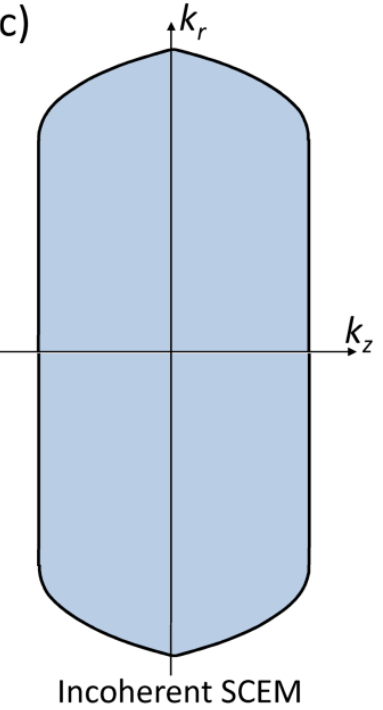

BF-STEM
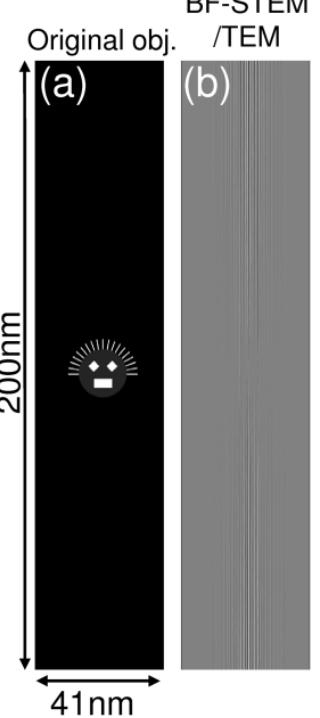

Coherent Incoherent

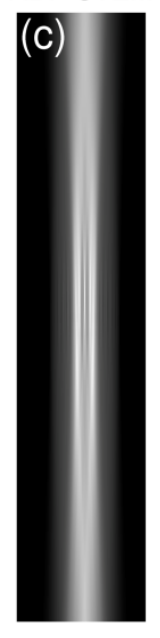

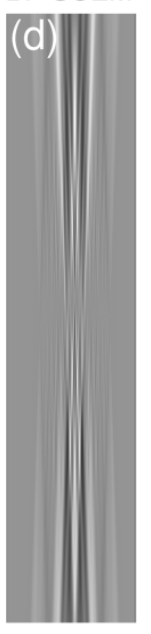

(e)

FIG. 1 (left one). Sketches of the analytic boundaries of the CTFs of (a) BF-STEM/TEM, (b) ADF-STEM and BF-SCEM (dashed line indicates HCI-ADF-STEM), (c) incoherent SCEM.

FIG. 2 (right one). Comparison of different imaging modes in depth-sectioning reconstruction $\left(200 \mathrm{KeV}, \alpha_{\max }=\right.$ $\left.40 \mathrm{mrad}, \mathrm{C}_{3}=-0.0235 \mathrm{~mm}, \mathrm{C}_{5}=14.70 \mathrm{~mm}, \mathrm{C}_{7}=0 \mathrm{~mm}, d f_{0}=-70.5 \AA\right):(\mathrm{a})$ the cross sectioning of the original object. The object is infinitely long in the direction perpendicular to the paper; (a-f) 3D reconstruction by (b) BF-STEM, (c) ADF-STEM, (d) coherent BF-STEM and (e) incoherent SCEM.

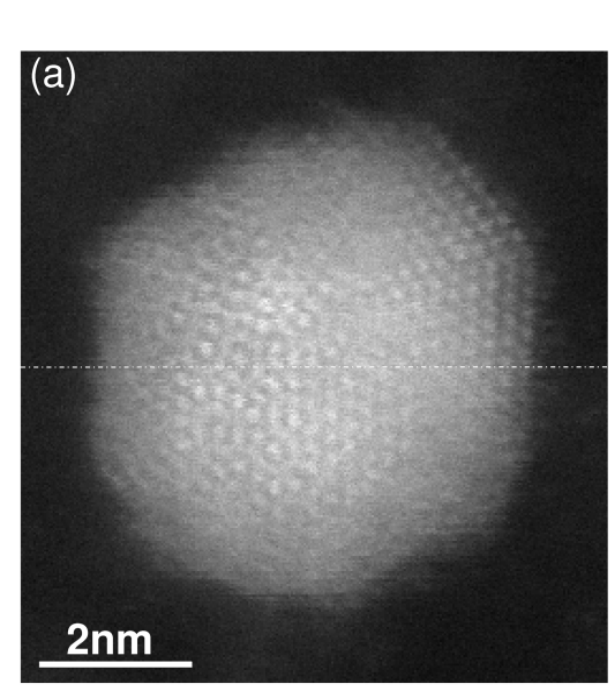

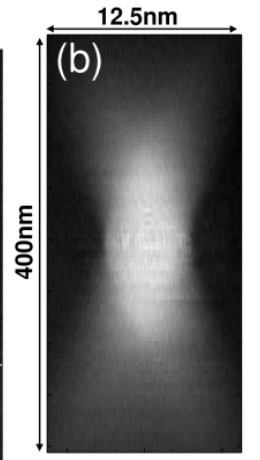
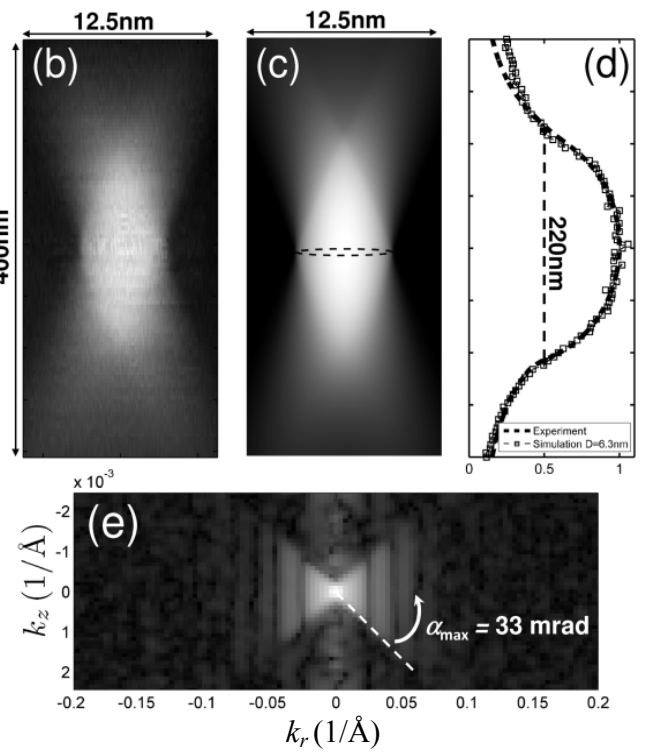

FIG. 3. Depth sectioning reconstruction of a $6 \mathrm{~nm}$ gold particle: (a) central slice of the gold particle. (b) the central cross section of the reconstruction; (c) the simulated reconstruction of a $6.3 \mathrm{~nm}$ solid sphere (the black dashed line outlines the original solid sphere); (d) the comparison of the central vertical line profile; (e) the central cross section of the 3D diffractogram of a $5.6 \mathrm{~nm}$ gold particle reconstruction. 